\title{
Modelling the chemistry and transport of bromoform within a sea breeze driven convective system during the SHIVA Campaign
}

P. D. Hamer ${ }^{1}$, V. Marécal ${ }^{1}$, R. Hossaini ${ }^{2}$, M. Pirre ${ }^{3}$, N. Warwick ${ }^{4}$, M. Chipperfield ${ }^{2}$, A. A. Samah ${ }^{5,6}$, N. Harris ${ }^{4}$, A. Robinson ${ }^{4}$, B. Quack ${ }^{7}$, A. Engel ${ }^{8}$, K. Krüger ${ }^{7}$, E. Atlas ${ }^{9}$, K. Subramaniam ${ }^{10}$, D. Oram ${ }^{11}$, E. Leedham ${ }^{11}$, G. Mills ${ }^{11}$, K. Pfeilsticker ${ }^{12}$, S. Sala ${ }^{8}$, T. Keber $^{8}$, H. Bönisch ${ }^{8}$, L. K. Peng ${ }^{13}$, M. S. M. Nadzir ${ }^{14}$, P. T. Lim $^{15}$, A. Mujahid ${ }^{15}$, A. Anton ${ }^{16}$, H. Schlager ${ }^{17}$, V. Catoire $^{3}$, G. Krysztofiak ${ }^{3}$, S. Fühlbrügge ${ }^{7}, M$. Dorf ${ }^{12}$, and W. T. Sturges ${ }^{11}$

${ }^{1}$ Centre National de Recherches Météorologiques-Groupe d'étude de l'Atmosphère Météorologique, Météo-France and CNRS, UMR3589, Toulouse, France

${ }^{2}$ The Institute for Climate \& Atmospheric, Science, School of Earth and Environment, University of Leeds, Leeds, UK

${ }^{3}$ Laboratoire de Physique et Chimie de l'Environnement et de l'Espace, CNRS and University of Orléans, UMR7328, Orléans, France

Modelling the chemistry and transport of bromoform

P. D. Hamer et al.

\section{Title Page}

$\triangleleft$

Back

\section{Close}

